\title{
PLANE WAVES NUMERICAL STABILITY OF SOME EXPLICIT EXPONENTIAL METHODS FOR CUBIC SCHRÖDINGER EQUATION* $^{*}$
}

\author{
Begoña Cano \\ Departamento de Matemática Aplicada, Universidad de Valladolid, IMUVA, Paseo de Belén, 7, 47011 \\ Valladolid, Spain.email: bego@mac.uva.es. \\ Adolfo González-Pachón \\ Departamento de Matemática Aplicada, Universidad de Valladolid, IMUVA, Paseo de Belén, 7, 47011 \\ Valladolid, Spain. email: adolfogp@mac.uva.es.
}

\begin{abstract}
Numerical stability when integrating plane waves of cubic Schrödinger equation is thoroughly analysed for some explicit exponential methods. We center on the following secondorder methods: Strang splitting and Lawson method based on a one-parameter family of 2-stage 2nd-order explicit Runge-Kutta methods. Regions of stability are plotted and numerical results are shown which corroborate the theoretical results. Besides, a technique is suggested to avoid the possible numerical instabilities which do not correspond to continuous ones.
\end{abstract}

Mathematics subject classification: 65M12,65M15, 65M99

Key words: numerical stability, exponential splitting Lawson methods, projection onto invariant quantities, plane waves, Schrödinger equation

\section{Introduction}

Numerical stability of plane wave solutions of cubic Schrödinger equation has already been a subject of research in the literature. In the first place, that analysis has been done in [11] for the first-order Lie splitting method when the initial condition is just a small perturbation of a constant. Secondly, in [6] the study has been performed for two implicit methods (Besse and Fei) for the more general case of small perturbations of initial conditions which have the form $u_{0}(x)=a e^{i k x}(a \in \mathbb{C}, k \in \mathbb{R}$.) In all previous cases, the analysis has been based on linear stability in the sense of ignoring terms which are quadratic on the perturbation. Afterwards, an analysis has been performed [8] using also modulated Fourier expansions for Strang splitting method. That type of analysis is theoretically valid for longer times although requires more restrictions on the parameters of integration. However, up to our knowledge, there is no numerical corroboration of the benefits of being more restrictive.

On the other hand, quite recently explicit exponential splitting and Runge-Kutta-based Lawson methods $[2-5,9]$ have been thoroughly developed and recommended for cubic Schrödinger equation. The former conserve two invariants (norm and momentum) while the latter do not. However, in [3-5], the conclusions are that, after projecting (very cheaply) on one of the invariants (norm), we are also projecting onto another invariant (momentum) for many solutions. Besides, plane wave solutions are among those. In the comparison with splitting methods in terms of computational efficiency [5], high order of accuracy in time and space is in favour

\footnotetext{
* Received ???? ??, 200? / Revised version received ???? ??, 200? / Accepted ???? ??, 200? /
} 
of projected Lawson methods, although exponential splitting methods are also many times a preferable tool.

The aim of this paper is to analyse and compare both type of methods with respect to its behaviour in terms of numerical stability when integrating plane wave solutions. For that, we will center for simplicity and, as a first stage, on second order methods: Strang splitting method [12] and Lawson methods based on explicit 2-stage Runge-Kutta methods. The latter is a one-parameter family of methods and all the analysis will be made in terms of that parameter (d). Besides, we will consider the unprojected and projected variants of these methods. In the numerical experiments, we have chosen pseudospectral discretization because it is very accurate for regular solutions and because it fits perfectly with the analysis in terms of the different frequencies which is done throughout the paper for the continuous in space problem.

We will see that Strang splitting method exactly integrates in time exact plane wave solutions (i.e without considering rounding errors). Nevertheless, Lawson methods do not. However, when projecting onto the norm, we achieve that the error not only in the momentum but also in the Hamiltonian vanishes for these solutions, which led us to believe that projected methods would behave better in terms of stability.

On the one hand, one conclusion in the paper is that the results are independent of the value of the frequency $k$ of the unperturbed wave in contrast with what happens with Besse \& Fei methods in [6]. On the other hand, in the comparison among Strang and Lawson methods, when $|\lambda||a|^{2}$ is small enough ( $\lambda$ being a real parameter in the equation), all methods behave in a similar manner. However, when $|\lambda||a|^{2}$ is bigger, Strang method behaves better than projected Lawson integrator and the last one better than the unprojected one.

In any case, we also suggest a filtering technique so as to try to avoid the numerical instabilities with all numerical methods when the continuous problem is stable.

The paper is structured as follows. Section 2 gives some preliminaries on the continuous problem and the considered numerical integrators. In Section 3, the behaviour of all considered methods when integrating the exact plane wave is justified. Besides, the precise results on the numerical stability with all methods when integrating a plane wave are stated. For the sake of clarity, the proofs have been relegated to an appendix. In Section 4, the different regions of stability are plotted for Strang and Lawson methods corresponding to $d=1$. Finally, in Section 5 the numerical performance is shown for the different methods, different initial conditions and different time stepsizes and the technique to avoid instabilities is suggested.

\section{Preliminaries}

\subsection{Continuous problem}

We will consider the equation

$$
u_{t}=i u_{x x}-i \lambda|u|^{2} u, \quad \lambda \in \mathbb{R},
$$


with periodic boundary conditions in a certain interval, which we will take as $[0,2 \pi]$ for the sake of simplicity. It is well known that this problem has as invariant quantities

$$
\begin{aligned}
& H=\frac{1}{2} \int_{0}^{2 \pi}\left(\left|u_{x}\right|^{2}+\frac{\lambda}{2}|u|^{4}\right), \\
& I_{1}=-\frac{1}{2} \int_{0}^{2 \pi}|u|^{2}, \\
& I_{2}=-\frac{1}{2} \int_{0}^{2 \pi} \operatorname{Im}\left(u \bar{u}_{x}\right),
\end{aligned}
$$

which are usually denoted by Hamiltonian, norm and momentum.

Plane wave solutions have the following form

$$
u(x, t)=a e^{i(k x-\omega t)}, \quad a \in \mathbb{C},
$$

where

$$
\omega=k^{2}+\lambda|a|^{2} .
$$

We are interested in studying the numerical stability when integrating these solutions. But let us first remind what happens in the continuous case [1]. If

$$
u_{0}=a\left(1+\sum_{l=-\infty}^{\infty} \hat{\epsilon}_{l}(0) e^{i l x}\right) e^{i k x}
$$

where all $\hat{\epsilon}_{l}(0)$ are assumed to be small, the exact solution of (2.1) can be written as

$$
u(x, t)=a e^{i(k x-\omega t)}\left(1+\sum_{l=-\infty}^{\infty} \epsilon_{l}(t) e^{i l x}\right),
$$

where, neglecting terms of higher order on $\epsilon_{l}$,

$$
\frac{d}{d t}\left(\begin{array}{c}
\epsilon_{l} \\
\bar{\epsilon}_{-l}
\end{array}\right)=i G_{l}\left(\begin{array}{c}
\epsilon_{l} \\
\bar{\epsilon}_{-l}
\end{array}\right), \quad G_{l}=\left(\begin{array}{cc}
-l^{2}-2 k l-\lambda|a|^{2} & -\lambda|a|^{2} \\
\lambda|a|^{2} & l^{2}-2 k l+\lambda|a|^{2}
\end{array}\right) .
$$

From this, it can be deduced that $\epsilon_{0}(t)=\hat{\epsilon}_{0}(0) e^{-\lambda|a|^{2} i t}$, which remains in modulus constant with time. On the other hand, for $l \neq 0$, as the eigenvalues of $i G_{l}$ are

$$
\left(-2 k \pm \sqrt{l^{2}+2 \lambda|a|^{2}}\right) i l,
$$

the corresponding mode is unstable when

$$
l^{2}<-2 \lambda|a|^{2}
$$

Therefore, instability can only occur when $\lambda<0$.

\subsection{Description of the methods}

We will consider Strang splitting, which is given, at each time stepsize, by

$$
u_{1}=e^{i \frac{h}{2} \partial_{x x}} \Psi_{h}\left(e^{i \frac{h}{2} \partial_{x x}} u_{0}\right),
$$


where $e^{i t \partial_{x x}} u_{I}$ denotes the exact solution of

$$
u_{t}=i u_{x x}, \quad u(0)=u_{I},
$$

after time $t$ and $\Psi_{t}(\bar{u})$ denotes the exact solution of

$$
u_{t}=-i \lambda|u|^{2} u, \quad u(0)=u_{I},
$$

after the same time. Notice that, as noticed in [11], $|u|$ is an invariant of this equation, and therefore

$$
\Psi_{t}\left(u_{I}\right)=e^{-i \lambda t\left|u_{I}\right|^{2}} u_{I} .
$$

On the other hand, for any nonzero value of the parameter $d$, we will consider the exponential Lawson method which is constructed from the second-order Runge-Kutta method corresponding to Butcher tableau

$$
\begin{array}{c|cc}
0 & 0 & \\
d & d & 0 \\
\hline & \frac{2 d-1}{2 d} & \frac{1}{2 d}
\end{array} .
$$

(For simplicity on the results, we restrict ourselves to $d \in(0,1]$, which are in fact the most interesting values in practice.) The exponential Lawson method [10] then reads as follows

$$
\begin{aligned}
K & =e^{i d h \partial_{x x}} u_{0}-d h i \lambda e^{i d h \partial_{x x}}\left|u_{0}\right|^{2} u_{0} \\
u_{1} & =e^{i h \partial_{x x}} u_{0}-h i \lambda\left[\frac{2 d-1}{2 d} e^{i h \partial_{x x}}\left|u_{0}\right|^{2} u_{0}+\frac{1}{2 d} e^{i(1-d) h \partial_{x x}}|K|^{2} K\right] .
\end{aligned}
$$

We will also consider its projection onto the norm $I_{1}$, which is an invariant of the problem. In such a way, the method is given by $[4,5]$,

$$
\tilde{u}_{1}=\sqrt{\frac{\int\left|u_{0}\right|^{2}}{\int\left|u_{1}\right|^{2}}} u_{1} .
$$

\section{Theoretical results}

\subsection{Behaviour of the different methods when integrating the exact plane wave}

Let us assume, for the moment, that the initial condition is the exact plane wave solution, without perturbations

$$
u_{0}=a e^{i k x} .
$$

Then, it is easy to observe that Strang method produces the exact solution after one stepsize since

$$
\begin{aligned}
e^{i t \partial_{x x}} e^{i k x} & =e^{i\left(k x-k^{2} t\right)}, \\
\Psi_{s}\left(a e^{i\left(k x-k^{2} t\right)}\right) & =a e^{-i \lambda|a|^{2} s} e^{i\left(k x-k^{2} t\right)}
\end{aligned}
$$

which implies that

$$
\begin{aligned}
u_{1} & =e^{i \frac{h}{2} \partial_{x x}} \Psi_{h}\left(a e^{i\left(k x-k^{2} \frac{h}{2}\right)}\right)=e^{i \frac{h}{2} \partial_{x x}}\left(e^{-i \lambda h|a|^{2}} a e^{i\left(k x-k^{2} \frac{h}{2}\right)}\right) \\
& =a e^{-i \lambda h|a|^{2}} e^{i\left(k x-k^{2} h\right)}=a e^{i\left(k x-h\left(k^{2}+\lambda|a|^{2}\right)\right)}=a e^{i(k x-\omega h) .}
\end{aligned}
$$



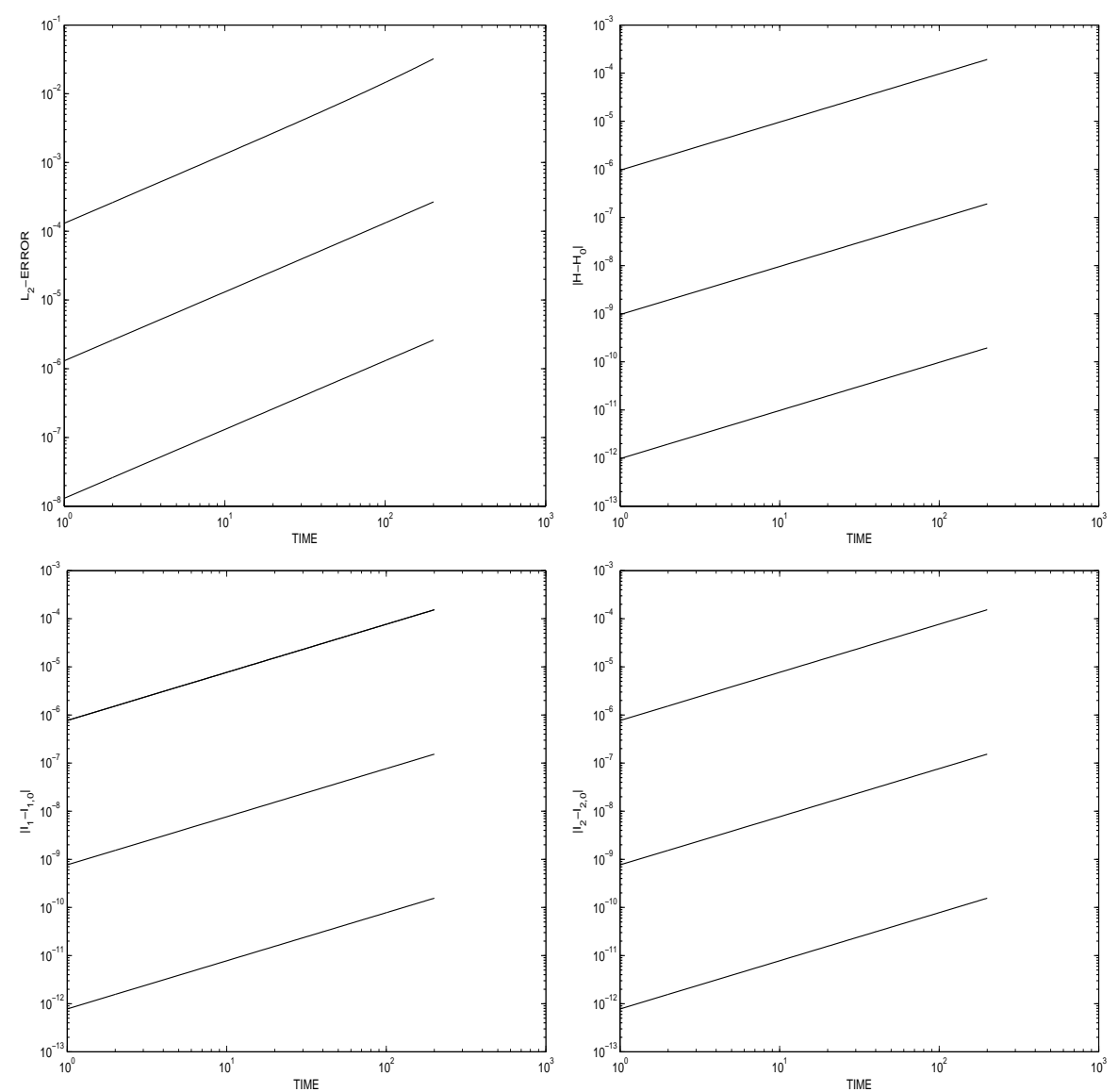

Fig. 3.1. Error against time with unprojected Lawson method $(d=1)$ when integrating an exact plane wave with $h=0.1,0.01,0.001: L^{2}$-error (top left), error in Hamiltonian (top right), norm error (bottom left), momentum error (bottom right)

On the other hand, the numerical solution after stepsize $h$ with Lawson method is given by

$$
u_{1}=a A e^{i(k x-\omega h)}
$$

where $\omega$ is given by $(2.3)$ and

$$
A=e^{i \lambda|a|^{2} h}\left(1-\frac{\lambda^{2} h^{2}}{2}|a|^{4}-\frac{\lambda^{4} h^{4}}{2} d^{2}|a|^{8}-\lambda h i|a|^{2}-\frac{\lambda^{3} h^{3} i}{2} d|a|^{6}\right) .
$$

It can be noticed that $A$ differs from 1 in

$$
h^{3} \lambda^{3}|a|^{6} i\left(\frac{d}{2}+\frac{1}{6}\right)+O\left(h^{4}\right)
$$

which corresponds to a local error behaving as $O\left(h^{3}\right)$ when $d \neq-\frac{1}{3}$ and leads in general to the second order for the global error in the $L^{2}$-norm. Nevertheless,

$$
|A|=1+\frac{1}{2} \lambda^{4} h^{4}|a|^{8}\left(-d^{2}+d+\frac{1}{4}\right)+O\left(h^{6}\right),
$$


which explains that the method shows order 3 in the invariants $I_{1}, I_{2}$ and $H(2.2)$, taking into account that

$$
\begin{aligned}
& |u(t)|^{2}=|a|^{2},\left|u_{1}\right|^{2}=|a|^{2}|A|^{2},\left|u_{x}(t)\right|^{2}=k^{2}|a|^{2},\left|u_{1, x}\right|^{2}=k^{2}|a|^{2}|A|^{2} \\
& u \bar{u}_{x}(t)=-i k|a|^{2}, u_{1} \bar{u}_{1, x}=-i k|a|^{2}|A|^{2} .
\end{aligned}
$$

This can be numerically corroborated in Figure 3.1 where that error is represented against time taking $N=10$ nodes in a pseudospectral space discretization and time stepsizes $h=$ $0.1,0.01,0.001$. We have considered (2.1) with $\lambda=1$ and initial condition $u_{0}=0.5 e^{i x}$. The value of the parameter $d$ has been taken as $d=1$.

However, when considering the projection of the method onto the norm $I_{1}[4,5]$, it happens that

$$
\tilde{u}_{1}=\sqrt{\frac{\int\left|u_{0}\right|^{2}}{\int\left|u_{1}\right|^{2}}} u_{1}=\frac{1}{|A|} u_{1}=a \frac{A}{|A|} e^{i(k x-\omega h)} .
$$

From this, it is clear that, with the projected method, not only the error in the norm vanishes but also the error in the momentum and the Hamiltonian.

Moreover, regarding stability, notice that the amplitude of the exact plane wave solution, before projecting and according to (3.5), is growing after a time stepsize when $d \in(0,1]$ for small enough $h$. However, after projecting, that does not happen any more, which seems to suggest that 'projecting' is going to be beneficial also for stability. The following results will be more precise in the behaviour for the perturbed plane wave solution.

\subsection{Behaviour of the different methods when integrating a perturbed plane wave}

In order to study the numerical stability when integrating a perturbed plane wave (2.4), we write the solution after stepsize $h$ as

$$
u_{1}=a\left(1+\sum_{l=-\infty}^{\infty} \hat{\epsilon}_{l, 1}(h) e^{i l x}\right) e^{i(k x-\omega h)} .
$$

After $n$ stepsizes we will consider the following notation

$$
u_{n}=a\left(1+\sum_{l=-\infty}^{\infty} \hat{\epsilon}_{l, n}(h) e^{i l x}\right) e^{i(k x-\omega n h)} .
$$

With our linear stability analysis, we neglect the terms of second order in the perturbation. This is not so serious if the perturbation is small and if we recognize that the results are valid for moderate times, but not too long times. At the same time, the results in [7] indicate that, in spite of the fact that the equation is nonlinear, when the perturbation of the plane wave is small enough in a Sobolev norm, the solution remains essentially localized in the same $k$-mode over very long times and the perturbation remains small in the same Sobolev norm. Although this result of stability is just for the continuous problem (and small enough amplitude of the plane wave solution when $\lambda<0$ ), it also helps the numerical method in terms of stability and in not mixing frequencies.

In fact, neglecting terms of second-order on $\hat{\epsilon}_{j}(0)$, the coefficients $\hat{\epsilon}_{l, n}(h)$ will just depend on those corresponding to the same frequency $\hat{\epsilon}_{l}(0)$. We are interested in looking at the growth of the former coefficients with respect to the latter and $n$, and we will say that the frequency $l$ is stable if $\hat{\epsilon}_{l, n}(h)$ does not grow with $n$. 
Theorem 3.1. When using Strang splitting, discarding the terms which are of quadratic order on the initial perturbations $\left\{\hat{\epsilon}_{j}(0)\right\}$,

$$
\left(\begin{array}{c}
\hat{\epsilon}_{l, 1}(h) \\
\overline{\hat{\epsilon}}_{-l, 1}(h)
\end{array}\right)=\left(\begin{array}{cc}
B_{l} & C_{l} \\
\bar{C}_{-l} & \bar{B}_{-l}
\end{array}\right)\left(\begin{array}{c}
\hat{\epsilon}_{l}(0) \\
\overline{\hat{\epsilon}}_{-l}(0)
\end{array}\right),
$$

where

$$
B_{l}=\left(1-i \lambda h|a|^{2}\right) e^{-\left(2 k l+l^{2}\right) h i}, \quad C_{l}=-i \lambda h|a|^{2} e^{-2 k l h i}
$$

From this,

$$
\left(\begin{array}{c}
\hat{\epsilon}_{l, n}(h) \\
\overline{\hat{\epsilon}}_{-l, n}(h)
\end{array}\right)=\left(\begin{array}{cc}
B_{l} & C_{l} \\
\bar{C}_{-l} & \bar{B}_{-l}
\end{array}\right)^{n}\left(\begin{array}{c}
\hat{\epsilon}_{l}(0) \\
\overline{\hat{\epsilon}}_{l}(0)
\end{array}\right)
$$

It happens that the modulus of all the eigenvalues of the matrix in (3.6) are independent of $k$ and just depend on $\lambda h|a|^{2}$ and $l^{2} h$. Furthermore, when

$$
\left.\left|\cos \left(l^{2} h\right)-\lambda h\right| a\right|^{2} \sin \left(l^{2} h\right) \mid<1
$$

the eigenvalues are different and have unit modulus, while whenever

$$
\left.\left|\cos \left(l^{2} h\right)-\lambda h\right| a\right|^{2} \sin \left(l^{2} h\right) \mid>1
$$

one of the eigenvalues has modulus $>1$. Moreover,

- For $l=0, \hat{\epsilon}_{0, n}$ satisfies

$$
\operatorname{Re}\left(\hat{\epsilon}_{0, n}(h)\right)=\operatorname{Re}\left(\hat{\epsilon}_{0}(0)\right), \quad \operatorname{Im}\left(\hat{\epsilon}_{0, n}(h)\right)=\operatorname{Im}\left(\hat{\epsilon}_{0}(0)\right)-2 \lambda h|a|^{2} n \operatorname{Re}\left(\hat{\epsilon}_{0}(0)\right),
$$

which implies that the real part of $\hat{\epsilon}_{0, n}$ remains constant at each step while the imaginary part grows linearly if $\operatorname{Re}\left(\hat{\epsilon}_{0}(0)\right) \neq 0, \lambda \neq 0$ and $a \neq 0$.

- For $l \neq 0$ and small enough $h$,

- Whenever $\lambda>0$, all frequencies are stable since all eigenvalues of the matrix in (3.6) are different and have unit modulus.

- Whenever $\lambda<0$, if $l^{2}<-2 \lambda|a|^{2}$, some eigenvalue of the matrix in (3.6) has modulus $>1$ (in fact $1+O(h))$, which makes those frequencies unstable. If $l^{2}>-2 \lambda|a|^{2}$, all eigenvalues are different and have unit modulus and are thus stable.

Remark 3.1. Notice that, for $k=0$, the conclusions about stability for Strang method are the same as those obtained for Lie-splitting method in Section 5.3 in [6], although the matrix in (3.6) is not the same.

Remark 3.2. Notice that, for small enough $h$, instability with Strang method only takes place when $\lambda<0$ and $l^{2}<-2 \lambda|a|^{2}$, which corresponds to the instability in the continuous problem. 
Theorem 3.2. When using Lawson methods (2.5), discarding the terms which are of quadratic order on the initial perturbations $\left\{\hat{\epsilon}_{j}(0)\right\}$, for $j \neq 0$, (3.6) applies with

$$
\begin{aligned}
B_{l}= & \left(1-2 \lambda h i|a|^{2}-\lambda^{3} h^{3} i d|a|^{6}-2 \lambda^{2} h^{2}|a|^{4}-2 \lambda^{4} h^{4} d^{2}|a|^{8}\right) e^{-\left(l^{2}+2 k l-\lambda|a|^{2}\right) h i} \\
& +\left(\frac{1}{2}\left(\lambda^{2} h^{2}|a|^{4}-\lambda^{4} h^{4} d^{2}|a|^{8}\right)-i \lambda^{3} h^{3} d|a|^{6}\right) e^{\left((2 d-1) l^{2}-2 k l+\lambda|a|^{2}\right) h i}, \\
C_{l}= & \left(\left(-\lambda^{4} h^{4} d^{2}|a|^{8}-\lambda^{2} h^{2}|a|^{4}\right)-\frac{\lambda h i}{2 d}(2 d-1)|a|^{2}\right) e^{-\left(l^{2}+2 k l-\lambda|a|^{2}\right) h i} \\
& +\left(-\lambda^{4} h^{4} d^{2}|a|^{8}-\frac{\lambda h i}{2 d}\left(|a|^{2}+3 \lambda^{2} h^{2} d^{2}|a|^{6}\right)\right) e^{\left((2 d-1) l^{2}-2 k l+\lambda|a|^{2}\right) h i} .
\end{aligned}
$$

Moreover, for $l=0$,

$$
\left(\begin{array}{c}
\hat{\epsilon}_{0,1}(h) \\
\overline{\hat{\epsilon}}_{0,1}(h)
\end{array}\right)=S_{0}\left(\begin{array}{c}
\hat{\epsilon}_{0}(0) \\
\overline{\hat{\epsilon}}_{0}(0)
\end{array}\right)+\left(\begin{array}{c}
A-1 \\
\bar{A}-1
\end{array}\right), \quad S_{0}=\left(\begin{array}{cc}
B_{0} & C_{0} \\
\bar{C}_{0} & \bar{B}_{0}
\end{array}\right)
$$

where $B_{0}$ and $C_{0}$ correspond to (3.10) and (3.11) with $l=0$ and $A$ to (3.3). From here, for $l \neq 0$ (3.8) applies again while for $l=0$,

$$
\begin{aligned}
\left(\begin{array}{c}
\hat{\epsilon}_{0, n}(h) \\
\hat{\epsilon}_{0, n}(h)
\end{array}\right)= & S_{0}^{n}\left[\left(\begin{array}{c}
\hat{\epsilon}_{0}(0) \\
\overline{\hat{\epsilon}}_{0}(0)
\end{array}\right)-\left(I-S_{0}\right)^{-1}\left(\begin{array}{c}
A-1 \\
\bar{A}-1
\end{array}\right)\right] \\
& +\left(I-S_{0}\right)^{-1}\left(\begin{array}{c}
A-1 \\
\bar{A}-1
\end{array}\right),
\end{aligned}
$$

where the last term (or the second one inside the brackets) can be seen to be $O\left(h^{2}\right)$.

It happens that, for all frequency $l$, the eigenvalues of matrix

$$
\left(\begin{array}{cc}
B_{l} & C_{l} \\
\bar{C}_{-l} & \bar{B}_{-l}
\end{array}\right)
$$

are independent of $k$ and just depend on $\lambda h|a|^{2}, h^{2} l$ and obviously $d$. On the other hand, for small enough $h$,

- Frequency $l=0$ is unstable since in this case all eigenvalues of the matrix which is raised to $n$ in (3.13) have modulus $>1$ (although $1+O\left(h^{4}\right)$ ).

- For $l \neq 0$,

- Whenever $\lambda>0$, the eigenvalues of matrix (3.14) have modulus $<1$ for $l^{2} \notin I$ with

$$
I=\left(\max \left\{0, \lambda|a|^{2}\left(1-\sqrt{\frac{-3 d^{2}+3 d+\frac{9}{4}}{2 d^{2}-2 d+1}}\right)\right\}, \lambda|a|^{2}\left(1+\sqrt{\frac{-3 d^{2}+3 d+\frac{9}{4}}{2 d^{2}-2 d+1}}\right)\right),
$$

while for $l^{2} \in I$, there exists at least an eigenvalue of modulus $>1\left(\right.$ in fact $\left.1+O\left(h^{4}\right)\right)$.

- Whenever $\lambda<0$, if $2 \lambda|a|^{2}+l^{2}<0$, frequency $l$ is unstable since there exists at least an eigenvalue of matrix (3.14) which has modulus $>1$ (in fact $1+O(h)$ ). If $2 \lambda|a|^{2}+l^{2}>0$, the eigenvalues have modulus $<1$ and the frequency is stable. 
Remark 3.3. Notice that, in contrast with Strang method, for small enough $h$, there appear instabilities with Lawson methods which do not correspond to the continuous ones. However, in that case, those instabilities are much weaker than those corresponding to the continuous problem. (The modulus of the eigenvalues is $1+O\left(h^{4}\right)$ against $1+O(h)$.) In practice, many times the former do not mean a real instability.

Theorem 3.3. When using projected Lawson methods (2.6), discarding the terms which are of quadratic order on the initial perturbations $\left\{\hat{\epsilon}_{j}(0)\right\}$, for $j \neq 0$,

$$
\left(\begin{array}{c}
\hat{\epsilon}_{l, 1}(h) \\
\overline{\hat{\epsilon}}_{-l, 1}(h)
\end{array}\right)=\frac{1}{|A|}\left(\begin{array}{cc}
B_{l} & C_{l} \\
\bar{C}_{-l} & \bar{B}_{-l}
\end{array}\right)\left(\begin{array}{c}
\hat{\epsilon}_{l}(0) \\
\overline{\hat{\epsilon}}_{-l}(0)
\end{array}\right),
$$

where $A$ is that in (3.3) and $B_{l}, C_{l}$ are those in (3.10)-(3.11). Moreover, for $l=0$,

$$
\left(\begin{array}{c}
\hat{\epsilon}_{0,1}(h) \\
\overline{\hat{\epsilon}}_{0,1}(h)
\end{array}\right)=\tilde{S}_{0}\left(\begin{array}{c}
\hat{\epsilon}_{0}(0) \\
\overline{\hat{\epsilon}}_{0}(0)
\end{array}\right)+\left(\begin{array}{c}
\frac{A}{|A|}-1 \\
\bar{A}-1 \\
\frac{|A|}{|A|}-1
\end{array}\right), \quad \tilde{S}_{0}=\left(\begin{array}{cc}
\tilde{B}_{0} & \tilde{C}_{0} \\
\overline{\tilde{C}}_{0} & \overline{\tilde{B}}_{0}
\end{array}\right),
$$

with

$$
\begin{aligned}
\tilde{B}_{0} & =\frac{A}{2|A|}\left(1-\frac{A \bar{C}_{0}+\bar{A} B_{0}}{|A|^{2}}\right)+\frac{B_{0}}{|A|}, \\
\tilde{C}_{0} & =\frac{A}{2|A|}\left(1-\frac{A \bar{B}_{0}+\bar{A} C_{0}}{|A|^{2}}\right)+\frac{C_{0}}{|A|} .
\end{aligned}
$$

From here, for $l \neq 0$ (3.8) applies again while for $l=0$,

$$
\begin{aligned}
\left(\begin{array}{c}
\tilde{\hat{\epsilon}}_{0, n}(h) \\
\tilde{\hat{\epsilon}}_{0, n}(h)
\end{array}\right)= & \tilde{S}_{0}^{n}\left[\left(\begin{array}{c}
\hat{\epsilon}_{0}(0) \\
\overline{\hat{\epsilon}}_{0}(0)
\end{array}\right)-\left(I-\tilde{S}_{0}\right)^{-1}\left(\begin{array}{c}
\frac{A}{|A|}-1 \\
\frac{A}{|A|}-1
\end{array}\right)\right] \\
& +\left(I-\tilde{S}_{0}\right)^{-1}\left(\begin{array}{c}
\frac{A}{|A|}-1 \\
\frac{A}{|A|}-1
\end{array}\right)
\end{aligned}
$$

where the last term (or the second one inside the brackets) can be seen to be $O\left(h^{2}\right)$.

It happens that $A$ and the modulus of the eigenvalues of the matrices in (3.16) and (3.17) are independent of $k$ and just depend on $\lambda h|a|^{2}, h^{2} l$ and obviously $d$. On the other hand, for small enough $h$,

- Frequency $l=0$ is stable since the matrix in (3.17) has all its eigenvalues of modulus $<1$.

- For $l \neq 0$,

- Whenever $\lambda>0$, the eigenvalues of

$$
\frac{1}{|A|}\left(\begin{array}{cc}
B_{l} & C_{l} \\
\bar{C}_{-l} & \bar{B}_{-l}
\end{array}\right)
$$

have modulus $<1$ for $l^{2} \notin \tilde{I}$ with

$$
\tilde{I}=\left(\max \left\{0, \lambda|a|^{2}\left(1-\sqrt{\frac{-2 d^{2}+2 d+2}{2 d^{2}-2 d+1}}\right)\right\}, \lambda|a|^{2}\left(1+\sqrt{\frac{-2 d^{2}+2 d+2}{2 d^{2}-2 d+1}}\right)\right),
$$

while for $l^{2} \in \tilde{I}$ there exists at least an eigenvalue of modulus $>1$ (in fact $1+O\left(h^{4}\right)$ ). 
- Whenever $\lambda<0$, if $2 \lambda|a|^{2}+l^{2}<0$, frequency $l$ is unstable since there exists at least an eigenvalue of (3.19) which has modulus $>1$ (in fact $1+O(h)$ ). If $2 \lambda|a|^{2}+l^{2}>0$, that frequency is stable since all eigenvalues have modulus $<1$.

Remark 3.4. Projecting Lawson method has improved stability for small enough $h$ in the following sense. On the one hand, for $l=0$, the weak instability which existed has disappeared. On the other hand, for $l \neq 0$ and $\lambda>0$, the set of values $\left\{l^{2}\right\}$ for which instability occurs diminishes since

$$
\sqrt{\frac{-2 d^{2}+2 d+2}{2 d^{2}-2 d+1}}<\sqrt{\frac{-3 d^{2}+3 d+\frac{9}{4}}{2 d^{2}-2 d+1}},
$$

for $d \in(0,1]$. In any case, we remark again that the possible instability is a very weak one since the modulus of the eigenvalues continues to be $1+O\left(h^{4}\right)$.

\section{Regions of stability}

In this section, we will observe when an unstable numerical behaviour will turn up. We are not just interested in what happens when $h \rightarrow 0$ for each particular $l$, but in what happens in a particular problem where several frequencies are important and we are considering a certain value of $h$ which is assumed to be good enough for consistency in the numerical integration of the problem.

As it was stated in previous theorems, for every method considered in this paper, the stability or instability just depends on the values $h \lambda|a|^{2}$ and $\sqrt{h} l$ for each frequency and it is independent of the value $k$ of the initial condition.

Therefore, by using coordinates $x=h \lambda|a|^{2}$ and $y=\sqrt{h} l$, we are plotting in the plane $(x, y)$ a dot where the matrices which determine stability in Theorems 3.1,3.2,3.3 happen to have an eigenvalue of modulus $>1.000001$ according to MATLAB. (We have chosen this value instead of 1 because of the high sensitivity of the calculus of the eigenvalues near double ones and because eigenvalues which differ in modulus from 1 less than 0.000001 do take a long time to show the unstable behaviour).

Figure 4.1 shows the regions of stability for Strang, unprojected Lawson method with $d=1$ and projected Lawson method corresponding also to $d=1$. In the considered area (the same as in [6]), the less unstable is clearly Strang method while the projected Lawson method is more stable than the unprojected one at least at $l=0$, which corroborates the results of previous sections. Notice also that the continuous instability corresponds to the interior of the parabola which is plotted in discontinuous red line in the figures.

One could think that all these methods are useless in front of the implicit ones considered in [6], where the regions of instability are much smaller. However, that is not the fact because, on the one hand, these methods are much cheaper because they are explicit, and on the other hand, the restriction on the stepsize because of consistency reasons leads to consider values of $h \lambda|a|^{2}$ much smaller than 30. (Notice for example that, for the exact plane wave solution, the local truncation error corresponding to the unprojected method (3.4) depends directly on the cube of such quantity). Therefore, in Figure 4.2, we have again plotted the regions of stability of the different methods but in a much narrower window on $h \lambda|a|^{2}$ than before. The conclusion is again clear. In that window, the area of instability of Strang method is quite smaller than that which corresponds to the projected and unprojected Lawson method, mainly when $\lambda>0$ 
and small $l$. The only clear improvement of the projected Lawson method over the unprojected one is that corresponding to the frequency $l=0$. Furthermore, notice that, for small enough $h$ and $\lambda<0$, the different regions of stability fit very well with the parabola which determines the continuous stability. This corroborates the results in the previous sections. More precisely, Theorems 3.1, 3.2 and 3.3. Similarly, for the unprojected and projected Lawson method, when $\lambda>0$, the regions of stability fit quite well with the parabolas $y^{2}=5 x / 2$ and $y^{2}=(1+\sqrt{2}) x$ respectively, which are drawn in discontinuous line in magenta and correspond to the upper extremes in the intervals $I$ and $\tilde{I}$ which determine the values of instability for $d=1$ in Theorems 3.2 and 3.3. We remark that in Figure 4.2 there is no fitting to such parabolas for the very smallest values of $h$ because we are drawing in blue just the values which lead to eigenvalues of modulus $>1+10^{-6}$. As the same theorems prove, the modulus of the eigenvalues in the region inside the parabola are $1+O\left(h^{4}\right)$ and therefore, for very small values of $h$, the program is not drawing them inside the instability region and, in fact, the instability would take a long time to be seen.

The following section will explain how to avoid, in any case, the instabilities corresponding to the higher frequencies.

\section{Avoiding instabilities}

In this section, we show how to avoid numerical instabilities when integrating perturbations of plane wave solutions of Schrödinger equation which satisfy that are regular or that have a very small but awkard noise. For that, we need to have a region of stability for the corresponding method which contains a small rectangle centered at $(0,0)$ in the variables $\left(h \lambda|a|^{2}, h^{1 / 2} l\right)$ except for the points inside the parabola which determines the continuous instability. Notice that, for Strang and the unprojected and projected Lawson method in the previous section, we could consider at least a rectangle of height $2 \times 1.5$ and width $2 \times 0.025$.

Notice that our problem (2.1) is very well fitted for pseudospectral discretization, so that the error in space can be considered negligible. We have integrated problem (2.1) with $\lambda=1$ and initial condition

$$
u_{0}=0.5\left(1+0.1 x^{7}(2 \pi-x)^{7} / \pi^{14}\right),
$$

which is a regular perturbation of the plane wave solution corresponding to $a=0.5$ and $k=0$. When considering $N=100$ nodes in space and time stepsizes $h=0.1,0.01,0.001$, we measure the error in the Hamiltonian and represent it against time in Figure 5.1 for the different methods considered. It is evident in all methods the instability which occurs when $h=0.1$ but which disappears when $h$ diminishes. For $l \neq 0$, this is in accordance with Theorems 3.2 and 3.3, as

$$
\lambda|a|^{2}\left(1+\sqrt{\frac{-3 d^{2}+3 d+\frac{9}{4}}{2 d^{2}-2 d+1}}\right)=5 / 8, \quad \lambda|a|^{2}\left(1+\sqrt{\frac{-2 d^{2}+2 d+2}{2 d^{2}-2 d+1}}\right)=\frac{1+\sqrt{2}}{4},
$$

in our problem and there is no value of $l^{2}$ with natural $l \neq 0$ which is less than any of those two values.

In Figure 5.2, we plot the maximum of the absolute value of the eigenvalues of the matrices which determine stability for $l=0,1, \ldots, 50$, for the different methods when $a=0.5, \lambda=1$ and $h=0.1$. (They are calculated with MATLAB again). It is clear that in all methods that maximum is mainly one except for $l=35$. We would also like to remark that, for $l=0$, for Strang method we obtain a maximum absolute value of $\sim 1+2 \times 10^{-12}$ while with the 
unprojected Lawson method we get $\sim 1+2 \times 10^{-7}$ and $\sim 1+9 \times 10^{-15}$ with the projected one. There is an improvement with the projection as it could be expected from Theorem 3.3. However, what causes the instability in all three methods is just the frequency $l=35$ in the range $[0: 1: 50]$. Even for the unprojected Lawson method, the numerical stability at $l=0$ is so weak that it is not seen in the experiments. For the other values of $h=0.01,0.001$, the maximum is always mainly less than 1 for that range of frequencies.

In order to better compare among the three methods considered in the paper, for the same problem we have taken as initial condition

$$
u_{0}=2\left(1+0.1 x^{7}(2 \pi-x)^{7} / \pi^{14}\right)
$$

which just means to change the value of $a$ to $a=2$. In such a way,

$$
\lambda|a|^{2}\left(1+\sqrt{\frac{-3 d^{2}+3 d+\frac{9}{4}}{2 d^{2}-2 d+1}}\right)=10, \quad \lambda|a|^{2}\left(1+\sqrt{\frac{-2 d^{2}+2 d+2}{2 d^{2}-2 d+1}}\right)=4(1+\sqrt{2}),
$$

so that for the unprojected and projected Lawson methods there exist values of $l \neq 0$ which lead to numerical instability for small $h$ when $\lambda>0$. Besides, although the modulus of one eigenvalue is just $1+O\left(h^{4}\right)$, the constant in $O\left(h^{4}\right)$ will contain a term of the form $C \lambda^{4}|a|^{8}$ for some moderate constant $C$. The fact that $\lambda=1$ and $a=2$ makes that term nonnegligible at least for not too smallest values of $h$. The same happens with the instability at $l=0$ for the unprojected method. In fact, for $h=0.1$, the maximum in modulus of the eigenvalues is calculated through MATLAB to be $\sim 1+3 \times 10^{-2}$ while it is $\sim 1+3 \times 10^{-7}$ for $h=0.01$. However, with projected Lawson method, the same value is exactly 1 for $h=0.1$ and $\sim 1+2 \times 10^{-15}$ for $h=0.01$. For Strang method, $\sim 1+8 \times 10^{-9}$ for $h=0.1$ and $\sim 1+2 \times 10^{-9}$ for $h=0.01$.

Figure 5.3 shows the errors with the three different methods, as before. All methods show instabilities although the unprojected Lawson method is the worst in the sense that it is not able to produce any result in the floating point arithmetic with $h=0.1$.

The maximum of the modulus of the eigenvalues is plotted in Figure 5.4 for $h=0.1$ and $h=0.01$ where the frequencies which produce instabilities can be observed in all cases. Except for $l=0$, that number of frequencies is very similar with the unprojected and projected Lawson methods. However, there are less frequencies which cause instability with Strang method.

What we suggest in this section, in order to avoid the possible instabilities with Strang and unprojected and projected Lawson method, is the following: At each step, when

$$
\sqrt{h}|l|>1.5
$$

if we denote by $C_{n}(l)$ the coefficient corresponding to the $l$ th-frequency in the pseudospectral discretization at step $n$, whenever $\left|C_{n+1}(l)\right|>1.000001\left|C_{n}(l)\right|$, we change $C_{n+1}(l)$ to

$$
\bar{C}_{n+1}(l)=C_{n+1}(l) \frac{\left|C_{n}(l)\right|}{\left|C_{n+1}(l)\right|},
$$

so that the coefficient corresponding to that frequency cannot grow with time in modulus. That is exactly what happens when the situation corresponds to stability in the continuous case. Besides, as we are not changing the coefficients of the smallest frequencies of the problem for small enough $h$ because of (5.1), the consistency of the methods is not altered for regular perturbations. (This is due to the fact that the coefficients of the bigger frequencies are much smaller than those of the smaller ones and therefore they are the ones which matter). The 
corresponding results, after filtering, are shown in Figure 5.5 for $a=0.5$ and in Figure 5.6 for $a=2$. For $a=0.5$, the improvement is clear with all methods. However, for $a=2$ and $h=0.1$ the unprojected Lawson method does not manage to get a solution and, with the projected one, the errors are still very bad. (The reason for that is that in that case $h \lambda|a|^{2}=0.4$, so that we are not in the 'rectangular' region of stability which was mentioned at the begining of the section.) Nevertheless, for Strang method and all considered timestepsizes and Lawson methods with the rest of values of $h$, the errors are already very much acceptable.

\section{A. Proof of Theorem 3.1}

Considering the particular form of $u_{0}$ (2.4) together with (3.1), neglecting terms of higher order in the small coefficients $\hat{\epsilon}_{j}(0)$, it can be shown that

$$
\begin{array}{r}
u_{1}=a e^{i\left(k x-\left(k^{2}+\lambda|a|^{2}\right) h\right)}\left[1+\left(1-i \lambda h|a|^{2}\right) \sum_{l=-\infty}^{\infty} \hat{\epsilon}_{l}(0) e^{-\left(2 k l+l^{2}\right) h i} e^{i l x}\right. \\
\left.-i \lambda h|a|^{2} \sum_{l=-\infty}^{\infty} \overline{\hat{\epsilon}}_{-l}(0) e^{-2 k l h i} e^{i l x}\right],
\end{array}
$$

from where, neglecting again terms of higher order on $\left\{\hat{\epsilon}_{j}(0)\right\},(3.6)$ is deduced with $B_{l}$ and $C_{l}$ in (3.7). To study the eigenvalues of this matrix, we must study the roots of

$$
z^{2}-\left(B_{l} \bar{B}_{-l}\right) z+B_{l} \bar{B}_{-l}-C_{l} \bar{C}_{-l} .
$$

Simple calculations give that

$$
\begin{aligned}
B_{l}+\bar{B}_{-l} & =2 e^{-2 k l h i}\left[\cos \left(l^{2} h\right)-\lambda h|a|^{2} \sin \left(l^{2} h\right)\right], \\
B_{l} \bar{B}_{-l}-C_{l} \bar{C}_{-l} & =e^{-4 k l h i},
\end{aligned}
$$

from what the eigenvalues of the matrix in (3.6) are the roots of

$$
\left[\frac{z}{e^{-2 k l h i}}\right]^{2}-2\left[\cos \left(l^{2} h\right)-\lambda h|a|^{2} \sin \left(l^{2} h\right)\right]\left[\frac{z}{e^{-2 k l h i}}\right]+1=0 .
$$

It is easy to deduce from here that when

$$
\left.\left|\cos \left(l^{2} h\right)-\lambda h\right| a\right|^{2} \sin \left(l^{2} h\right) \mid<1
$$

both roots are different and have unit modulus which implies that the perturbations on the corresponding coefficients propagate in a stable way. However, when

$$
\left.\left|\cos \left(l^{2} h\right)-\lambda h\right| a\right|^{2} \sin \left(l^{2} h\right) \mid>1,
$$

one of the roots will have modulus $>1$ and therefore, the corresponding perturbation will propagate in an unstable way. Finally, when

$$
\cos \left(l^{2} h\right)-\lambda h|a|^{2} \sin \left(l^{2} h\right)=1 \quad \text { or } \quad \cos \left(l^{2} h\right)-\lambda h|a|^{2} \sin \left(l^{2} h\right)=-1,
$$

both roots are the same and have modulus equal to 1 . Nevertheless, the matrix does not diagonallize if $a \neq 0$ and $\lambda \neq 0$, so the $n$ th-powers of the matrix in (3.6) grow linearly. This case corresponds, for example, to $l=0$, for which the the precise formula (3.9) is deduced. 
On the other hand, for $l \neq 0$, by considering the asymptotic expansion on $h$,

$$
\cos \left(l^{2} h\right)-\lambda h|a|^{2} \sin \left(l^{2} h\right)=1-l^{2} h^{2}\left(\frac{l^{2}}{2}+\lambda|a|^{2}\right)+O\left(h^{4}\right),
$$

from what, for small enough $h$, if $\lambda>0$, the absolute value of this is always $<1$ and all frequencies are stable. Nevertheless, if $\lambda<0$, whenever $l^{2}<-2 \lambda|a|^{2}$, the absolute value is $>1$ and the corresponding frequency is unstable while otherwise, it is stable. Notice also, to be more precise, that, in the unstable case, one of the roots satisfies

$$
\frac{z}{e^{-2 k l h i}}=1+h|l| \sqrt{-l^{2}-2 \lambda|a|^{2}}+O\left(h^{2}\right)
$$

from what the theorem is completely proved.

\section{B. Proof of Theorem 3.2}

It is just a question of patience to deduce from (2.5) that, when $u_{0}$ is like in (2.4), after neglecting terms of higher order on $\left\{\hat{\epsilon}_{j}(0)\right\}$,

$$
u_{1} \approx a\left[A+\sum_{l=-\infty}^{\infty} B_{l} \hat{\epsilon}_{l}(0) e^{i l x}+\sum_{l=-\infty}^{\infty} C_{l} \overline{\hat{\epsilon}}_{-l}(0) e^{i l x}\right] e^{i(k x-\omega h)}
$$

where $B_{l}$ and $C_{l}$ are those in (3.10)-(3.11).

From here, except for terms of higher order on $\left\{\hat{\epsilon}_{l}(0)\right\}$, for $l \neq 0,(3.6)$ and (3.8) apply with $B_{l}$ and $C_{l}$ in (3.10)-(3.11). Moreover, for $l=0,(3.12)$ and (3.13) are satisfied where the last term (or the second term inside the brackets) can be seen to be $O\left(h^{2}\right)$.

From (3.10) and (3.11),

$$
\begin{aligned}
& B_{0}=\left(1-\frac{3}{2} \lambda^{2} h^{2}|a|^{4}-\frac{5}{2} \lambda^{4} h^{4} d^{2}|a|^{8}+i\left(-2 \lambda h|a|^{2}-2 \lambda^{3} h^{3} d|a|^{6}\right)\right) e^{\lambda|a|^{2} h i}, \\
& C_{0}=\left(-\lambda^{2} h^{2}|a|^{4}-2 \lambda^{4} h^{4} d^{2}|a|^{8}-i\left(\lambda h|a|^{2}+\frac{3}{2} \lambda^{3} h^{3} d|a|^{6}\right)\right) e^{\lambda|a|^{2} h i} .
\end{aligned}
$$

Now, we will take into account that, by making the corresponding calculations,

$$
\begin{aligned}
B_{0} \bar{B}_{0} & -C_{0} \bar{C}_{0} \\
& =1+\left(\frac{5}{4}-5 d^{2}+5 d\right) \lambda^{4} h^{4}|a|^{8}-\frac{13}{4} \lambda^{6} h^{6} d^{2}|a|^{12}+\frac{9}{4} \lambda^{8} h^{8} d^{4}|a|^{16}
\end{aligned}
$$

from what

$$
\left(B_{0}+\bar{B}_{0}\right)^{2}-4\left(B_{0} \bar{B}_{0}-C_{0} \bar{C}_{0}\right)=\left(10 d^{2}-12 d-\frac{19}{6}\right) \lambda^{4} h^{4}|a|^{8}+O\left(h^{6}\right) .
$$

Now the expression in brackets in the $O\left(h^{4}\right)$-term is a parabola which takes negative values for $d \in(0,1]$, which implies that the eigenvalues of the matrix in (3.12) are complex conjugate. Now, for the same values of $d$, the expression in brackets in the term which is $O\left(h^{4}\right)$ in (B.4) is a parabola which is positive in the mentioned interval, which proves that the modulus of the eigenvalues is $>1$ for small enough $h$. 
On the other hand, for $l \neq 0$, by making the corresponding calculations, it happens that

$$
\begin{gathered}
B_{l}+\bar{B}_{-l}=e^{-2 k l h i}\left[2\left(1-2 \lambda^{2} h^{2}|a|^{4}-2 \lambda^{4} h^{4} d^{2}|a|^{8}\right) \cos \left(\left(l^{2}-\lambda|a|^{2}\right) h\right)\right. \\
-2\left(2 \lambda h|a|^{2}+\lambda^{3} h^{3} d|a|^{6}\right) \sin \left(\left(l^{2}-\lambda|a|^{2}\right) h\right) \\
+\left(\lambda^{2} h^{2}|a|^{4}-\lambda^{4} h^{4} d^{2}|a|^{8}\right) \cos \left(\left((2 d-1) l^{2}+\lambda|a|^{2}\right) h\right) \\
\left.+2 \lambda^{3} h^{3} d|a|^{6} \sin \left(\left((2 d-1) l^{2}+\lambda|a|^{2}\right) h\right)\right] . \\
B_{l} \bar{B}_{-l}-C_{l} \bar{C}_{-l} \quad \\
=e^{-4 k l h i\left[1-\frac{4 d^{2}-4 d+2}{4 d^{2}} \lambda^{2} h^{2}|a|^{4}+\left(\frac{7}{4}-4 d^{2}+4 d\right) \lambda^{4} h^{4}|a|^{8}\right.} \\
+\frac{21}{4} \lambda^{6} h^{6} d^{2}|a|^{12}+\frac{9}{4} \lambda^{8} h^{8} d^{4}|a|^{16} \\
+\left[\frac{4 d^{2}-4 d+2}{4 d^{2}} \lambda^{2} h^{2}|a|^{4}+\left(d-d^{2}-\frac{1}{2}\right) \lambda^{4} h^{4}|a|^{8}\right] \cos \left(2 d l^{2} h\right) \\
\left.-\frac{4 d-4 d^{2}-2}{2 d} \lambda^{3} h^{3}|a|^{6} \sin \left(2 d l^{2} h\right)\right] .
\end{gathered}
$$

We notice that the terms in brackets do not depend on $k$, just on $d, l^{2} h$ and $\lambda h|a|^{2}$. Expanding these terms till $O\left(h^{4}\right)$, the eigenvalues of (3.14) satisfy

$$
\frac{z}{e^{-2 k l h i}} \approx 1-\left(\lambda l^{2}|a|^{2}+\frac{l^{4}}{2}\right) h^{2} \pm \sqrt{1-\left(2 \lambda l^{2}|a|^{2}+l^{4}\right) h^{2}-1} .
$$

Now, if $2 \lambda|a|^{2}+l^{2}>0$, for small enough $h$,

$$
\frac{z}{e^{-2 k l h i}} \approx 1-\left(\lambda l^{2}|a|^{2}+\frac{l^{4}}{2}\right) h^{2} \pm i \sqrt{2 \lambda|a|^{2} l^{2}+l^{4}} h
$$

which has modulus 1 except for $O\left(h^{4}\right)$. As this is just an approximation of the eigenvalue, we must consider at least also the terms in $O\left(h^{4}\right)$ to discuss about stability. Besides, it is clear that, in this case of complex conjugate values, the square of the modulus of those values will be

$$
\frac{B_{l} \bar{B}_{-l}-C_{l} \bar{C}_{-l}}{e^{-4 k l h i}}
$$

The coefficient of $h^{4}$ in this expression can be proved to be, except for the factor $\lambda^{2}|a|^{4}$,

$$
-\left(2 d^{2}-2 d+1\right) l^{4}-\left(4 d-4 d^{2}-2\right) l^{2} \lambda|a|^{2}+\left(\frac{5}{4}-5 d^{2}+5 d\right) \lambda^{2}|a|^{4} .
$$

This can be interpreted as a parabola in $x=l^{2}$, which gets its maximum at $x=\lambda|a|^{2}$.

When $\lambda>0$, this value is positive. There, the parabola takes the value $\left(-3 d^{2}+3 d+\frac{9}{4}\right) \lambda^{2}|a|^{4}$. This number can be proved to be positive when $d \in(0,1]$. Besides, for those values of $d$, the parabola vanishes at

$$
x=\lambda|a|^{2}\left[1 \pm \sqrt{\frac{-3 d^{2}+3 d+\frac{9}{4}}{2 d^{2}-2 d+1}}\right] .
$$

As the rational function inside the square root can take values between 0 and 6 depending on the explicit value of $d$, the values of $l$ which lead to eigenvalues of modulus $>1$ for small enough $h$ are those which may lie in the range $l^{2} \in I$, with $I$ in (3.15). 
When $\lambda<0$, the abscissa of the maximum of the parabola $x=\lambda|a|^{2}$ is negative, Besides, the value of this parabola at $x=-2 \lambda|a|^{2}>0$ can be calculated to be

$$
\lambda^{2}|a|^{4}\left[-21 d^{2}+21 d-\frac{27}{4}\right]
$$

which can be proved to be negative for every real value $d$. From here, the parabola is always negative for $x>-2 \lambda|a|^{2}$. This means that, for small enough $h$, the eigenvalues of the studied matrix have modulus $<1$ for $l^{2}>-2 \lambda a^{2}$.

On the other hand, if $2 \lambda|a|^{2}+l^{2}<0$, from (B.5),

$$
\frac{z}{e^{-2 k l h i}} \approx 1-\left(\lambda l^{2}|a|^{2}+\frac{l^{4}}{2}\right) h^{2} \pm \sqrt{-\left(2 \lambda|a|^{2} l^{2}+l^{4}\right)} h
$$

and therefore, for small enough $h$, there is always an eigenvalue of modulus $>1$.

\section{Proof of Theorem 3.3}

Notice that, when $u_{0}$ has the general form (2.4), by using (B.1), the projected method after one stepsize gives

$$
\tilde{u}_{1}=\sqrt{\frac{\int\left|u_{0}\right|^{2}}{\int\left|u_{1}\right|^{2}}} u_{1}=\sqrt{\frac{1+\hat{\epsilon}_{0}(0)+\overline{\hat{\epsilon}}_{0}(0)}{|A|^{2}+\left(A \bar{B}_{0}+\bar{A} C_{0}\right) \overline{\hat{\epsilon}}_{0}(0)+\left(A \bar{C}_{0}+\bar{A} B_{0}\right) \hat{\epsilon}_{0}(0)}} u_{1} .
$$

From here, notice that

$$
\tilde{u}_{1}=a\left(1+\sum_{l=-\infty}^{\infty} \tilde{\hat{\epsilon}}_{l, 1}(h) e^{i l x}\right) e^{i k(x-\omega h)}
$$

where, neglecting terms of higher order in $\hat{\epsilon}_{j}(0)$,

$$
\left(\begin{array}{c}
\tilde{\hat{\epsilon}}_{0,1}(h) \\
\overline{\hat{\epsilon}}_{0,1}(h)
\end{array}\right)=\left(\begin{array}{ll}
\tilde{B}_{0} & \tilde{C}_{0} \\
\tilde{C}_{0} & \tilde{B}_{0}
\end{array}\right)\left(\begin{array}{c}
\hat{\epsilon}_{0}(0) \\
\overline{\hat{\epsilon}}_{0}(0)
\end{array}\right)+\left(\begin{array}{c}
\frac{A}{|A|}-1 \\
\frac{A}{|A|}-1
\end{array}\right),
$$

with

$$
\begin{aligned}
& \tilde{B}_{0}=\frac{A}{2|A|}\left(1-\frac{A \bar{C}_{0}+\bar{A} B_{0}}{|A|^{2}}\right)+\frac{B_{0}}{|A|}, \\
& \tilde{C}_{0}=\frac{A}{2|A|}\left(1-\frac{A \bar{B}_{0}+\bar{A} C_{0}}{|A|^{2}}\right)+\frac{C_{0}}{|A|} .
\end{aligned}
$$

Then, in the same way than (3.13), (3.18) can be deduced where the last term (or the second term inside the brackets) can be seen to be $O\left(h^{2}\right)$.

By making the corresponding calculations, it can be proved that

$$
\begin{gathered}
\tilde{B}_{0} \overline{\tilde{B}}_{0}-\tilde{C}_{0} \overline{\tilde{C}}_{0}=\frac{1}{|A|^{4}}\left[\left|B_{0}\right|^{2}|A|^{2}-\left|C_{0}\right|^{2}|A|^{2}+\operatorname{Re}\left(A \bar{B}_{0}\left(|A|^{2}-A \bar{C}_{0}-\bar{A} B_{0}\right)\right)\right. \\
\left.-\operatorname{Re}\left(A \bar{C}_{0}\left(|A|^{2}-A \bar{B}_{0}-\bar{A} C_{0}\right)\right)\right] .
\end{gathered}
$$

Then from (3.3), (B.2) and (B.3), it is deduced that

$$
\tilde{B}_{0} \overline{\tilde{B}}_{0}-\tilde{C}_{0} \overline{\tilde{C}}_{0}=1-\lambda^{6} h^{6}|a|^{12}\left(\frac{19}{4} d^{2}+\frac{3}{4}\right)+O\left(h^{8}\right) .
$$


As the coefficient of $h^{6}$ is always negative, this implies that the product of the eigenvalues of the corresponding matrix is always less than 1 for small enough $h$, independently of the value of $\lambda$ and $d$. Moreover,

$$
\left(\tilde{B}_{0}+\overline{\tilde{B}}_{0}\right)^{2}-4\left(\tilde{B}_{0} \overline{\tilde{B}}_{0}-\tilde{C}_{0} \overline{\tilde{C}}_{0}\right)=-4 \lambda^{4} h^{4}|a|^{8}\left(\frac{1}{3}+d\right)+O\left(h^{6}\right)
$$

As the coefficient of $h^{4}$ in this expression is negative for $d \in(0,1]$, the eigenvalues of the corresponding matrix are complex conjugate and, by (C.4), they have modulus $<1$.

Now, for $l \neq 0,(3.16)$ is clearly deduced and the modulus of the eigenvalues of (3.14) divided by $|A|$ must be studied.

From the proof of Theorem 3.2, in the case of complex conjugate roots $\left(2 \lambda|a|^{2}+l^{2}>0\right)$, to the square of the modulus of those roots we now have to multiply by $1 /|A|^{2}$, which is $1-\lambda^{4} h^{4}|a|^{8}\left(-d^{2}+d+\frac{1}{4}\right)$. In such a way, the coefficient of $h^{4}$ in that expression, except for the factor $\lambda^{2}|a|^{4}$, is

$$
-\left(2 d^{2}-2 d+1\right) l^{4}-\left(4 d-4 d^{2}-2\right) l^{2} \lambda|a|^{2}+\left(1-4 d^{2}+4 d\right) \lambda^{2}|a|^{4} .
$$

Again this can be interpreted as a parabola in $x=l^{2}$ which also gets its maximum at $x=\lambda|a|^{2}$.

When $\lambda>0$, the abscissa of the maximum is $>0$. That maximum is $\left(-2 d^{2}+2 d+2\right) \lambda^{2}|a|^{4}$, which is positive when $d \in(0,1]$. On the other hand, in the latter case, the parabola vanishes at

$$
\lambda|a|^{2}\left(1 \pm \sqrt{\frac{-2 d^{2}+2 d+2}{2 d^{2}-2 d+1}}\right)
$$

from what the result on the interval $\tilde{I}$ follows.

When $\lambda<0$, the abscissa of the maximum is $<0$. Now, the value of the parabola at $x=-2 \lambda|a|^{2}>0$ can be calculated to be

$$
\lambda^{2}|a|^{4}\left(-20 d^{2}+20 d-7\right),
$$

which is negative for every real value $d$. This implies that, for $l^{2}>-2 \lambda|a|^{2}$, the eigenvalues have modulus $<1$ for small enough $h$. On the other hand, for $l^{2}<-2 \lambda|a|^{2}$, there is at least an eigenvalue of modulus $>1$ considering (B.6) in the proof of Theorem 3.2 and the fact that the modulus $>1$ there is due to a term which is $O(h)$ and not $O\left(h^{4}\right)$, as the difference between 1 and $1 /|A|$.

Acknowledgments. This work has been supported by project MTM 2011-23417.

\section{References}

[1] G. P. Agrawal, Nonlinear Fiber Optics, 5th ed., Academic Press, 2013.

[2] H. Berland, A. L. Islas and C. M. Schober, Conservation of phase space properties using exponential integrators on the cubic Schrödinger equation, J. Comp. Phys. 225 (2007) 284-299.

[3] B. Cano and A. González-Pachón, Exponential methods for the time integration of Schrödinger equation, AIP Conference Proceedings Volume 1281 (2010), CDROM ISBN: 978-0-7354-0831-9.

[4] B. Cano and A. González-Pachón, Exponential time integration of solitary waves of cubic Schrödinger equation, Applied Numerical Mathematics 91 (2015) 26-45.

[5] B. Cano and A. González-Pachón, Projected explicit Lawson methods for the integration of Schrödinger equation, Numer. Meth. Part. Differ. Equat. 31 (2015) 78-104 .

[6] M. Dahlby and B. Owren, Plane wave stability of some conservative schemes for the cubic Schrödinger equation, ESAIM, Math. Model. and Numer. Anal. 43:4 (2009) 677-687. 
[7] E. Faou, L. Gauckler and C. Lubich, Sobolev stability of plane wave solutions to the cubic nonlinear Schrödinger equation on a torus, Comm. Partial Differential Equations 38 (2013) 1123-1140 .

[8] E. Faou, L. Gauckler and C. Lubich, Plane wave stability of the split-step Fourier method for the nonlinear Schrödinger equation, Forum of Mathematics, Sigma, Vol. 2, e5, 45 pages (2014) . DOI: $10.1017 /$ fms.2014.4

[9] O. Koch, Ch. Neuhaser and M. Thalhammer, Embedded Exponential Operator Splitting Methods for the Time Integration of Nonlinear Evolution Equations, Appl. Numer. Math. 63 (2013) 14-24.

[10] J. D. Lawson, Generalized Runge-Kutta processes for stable systems with large Lipschitz constants, SIAM J. Numer. Anal. 4 (1967) 372-380.

[11] J. A. C. Weideman and B. M. Herbst, Split-step methods for the solution of the nonlinear Schrödinger equation, SIAM J. Numer. Anal. 23 (1986) 485-507.

[12] G. Strang, On the construction and comparison of difference schemes, SIAM J. Numer. Anal. 5 (1968) 506-517. 

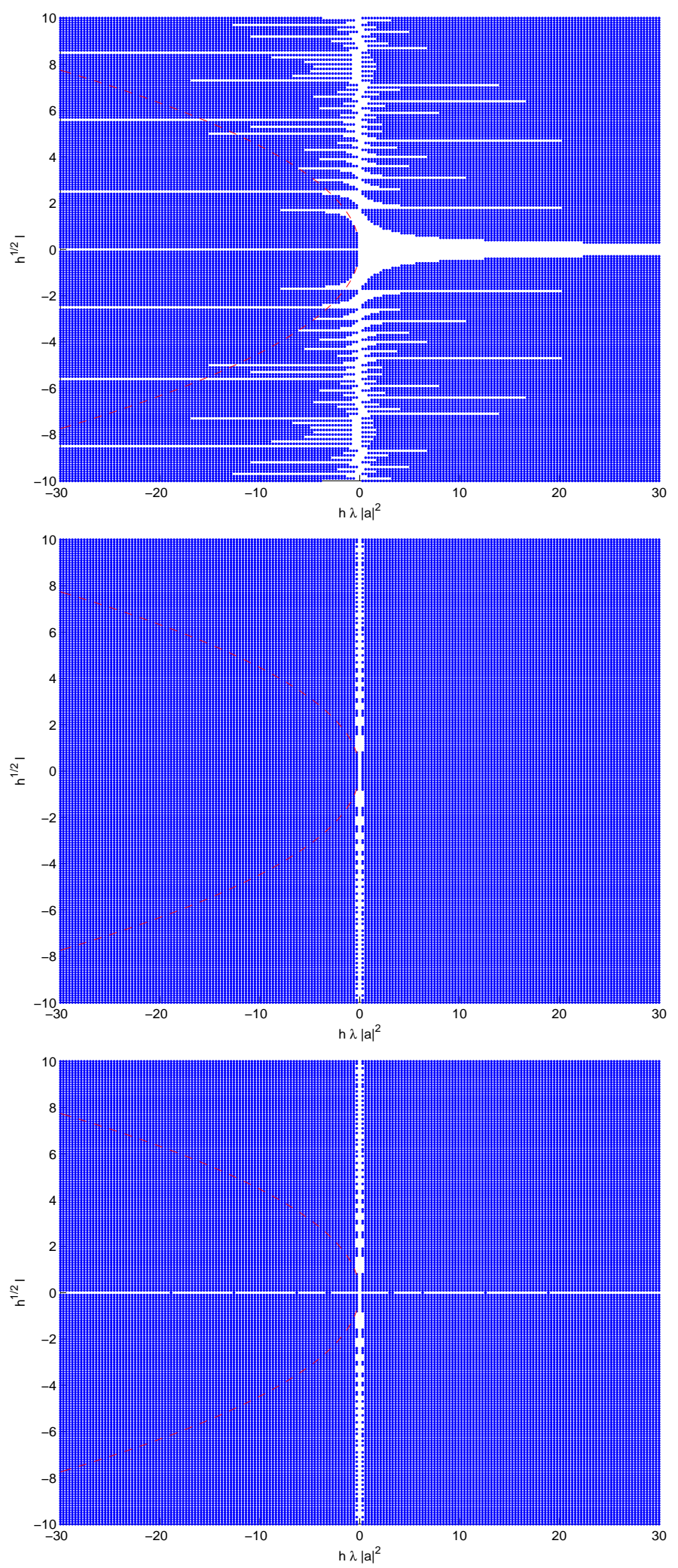

Fig. 4.1. Regions of stability for Strang method (top), Lawson method (d=1) when unprojected (middle) and projected (bottom) (blue corresponds to instability of the method and the interior of the parabola in red discontinuous line corresponds to continuous instability) 

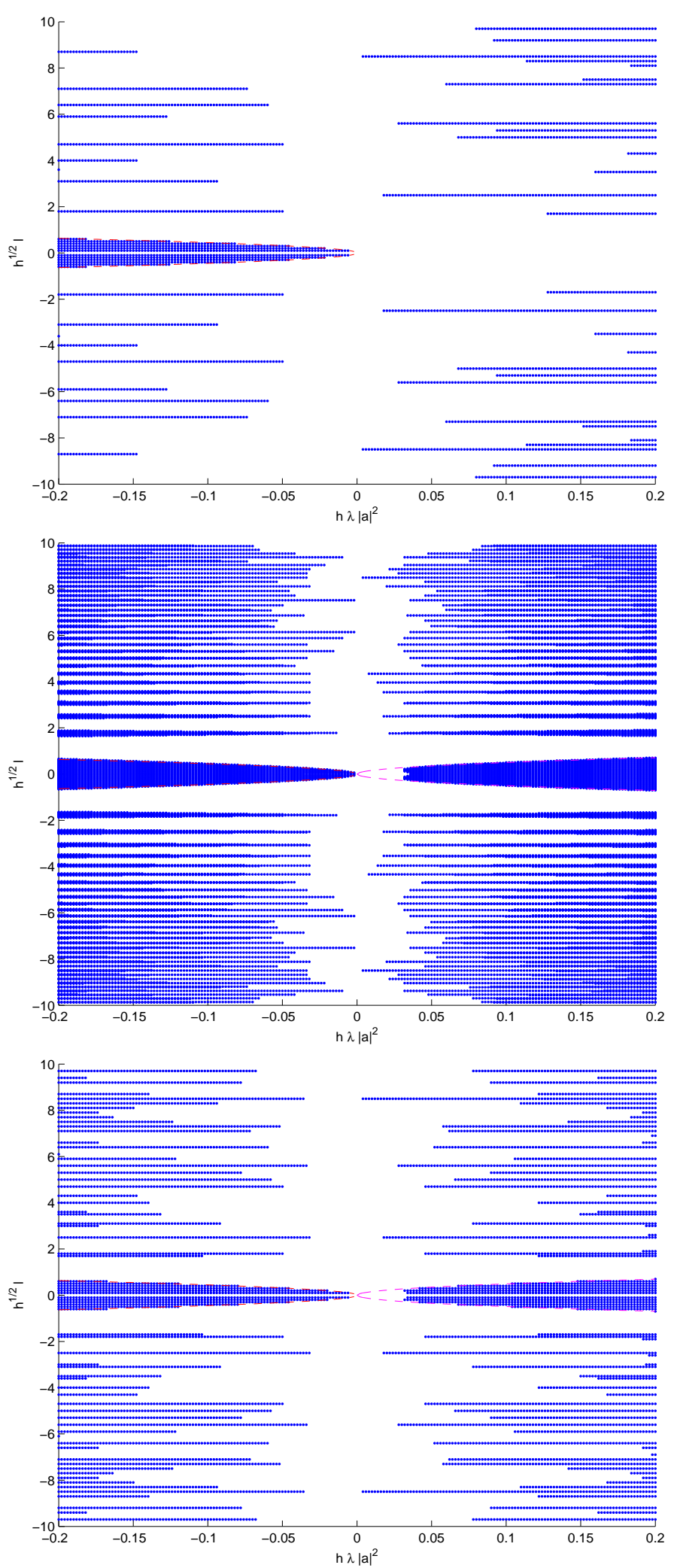

Fig. 4.2. Regions of stability for Strang method (top), Lawson method (d=1) when unprojected (middle) and projected (bottom), (blue corresponds to instability of the method and the interior of the parabola in red discontinuous line corresponds to continuous instability; the interior of the parabola in magenta discontinuous line corresponds to instability of the method for $\lambda>0$ and small enough $h$ through Theorems 3.4 and 3.6) 

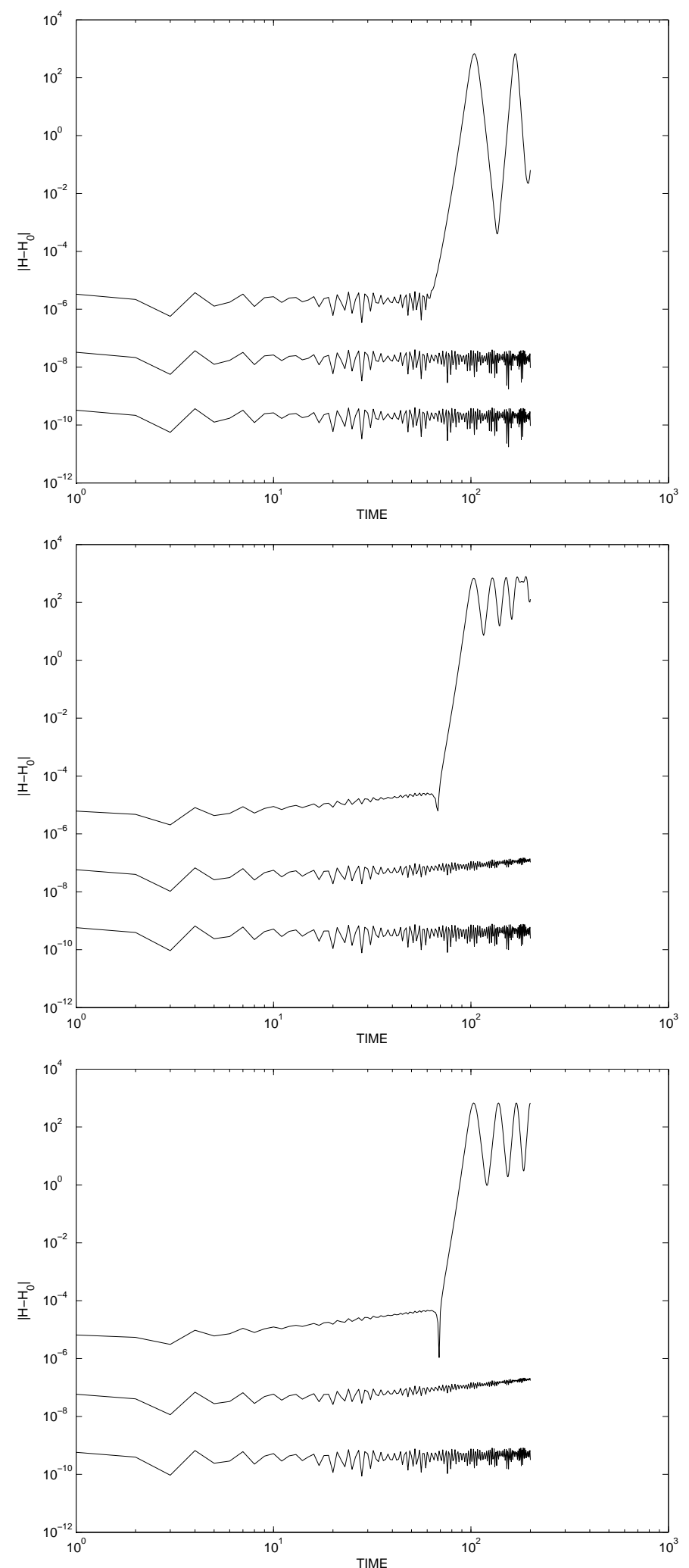

Fig. 5.1. Strang method (top), Lawson method $(\mathrm{d}=1)$ when unprojected (middle) and projected (bottom), when $a=1 / 2$. 

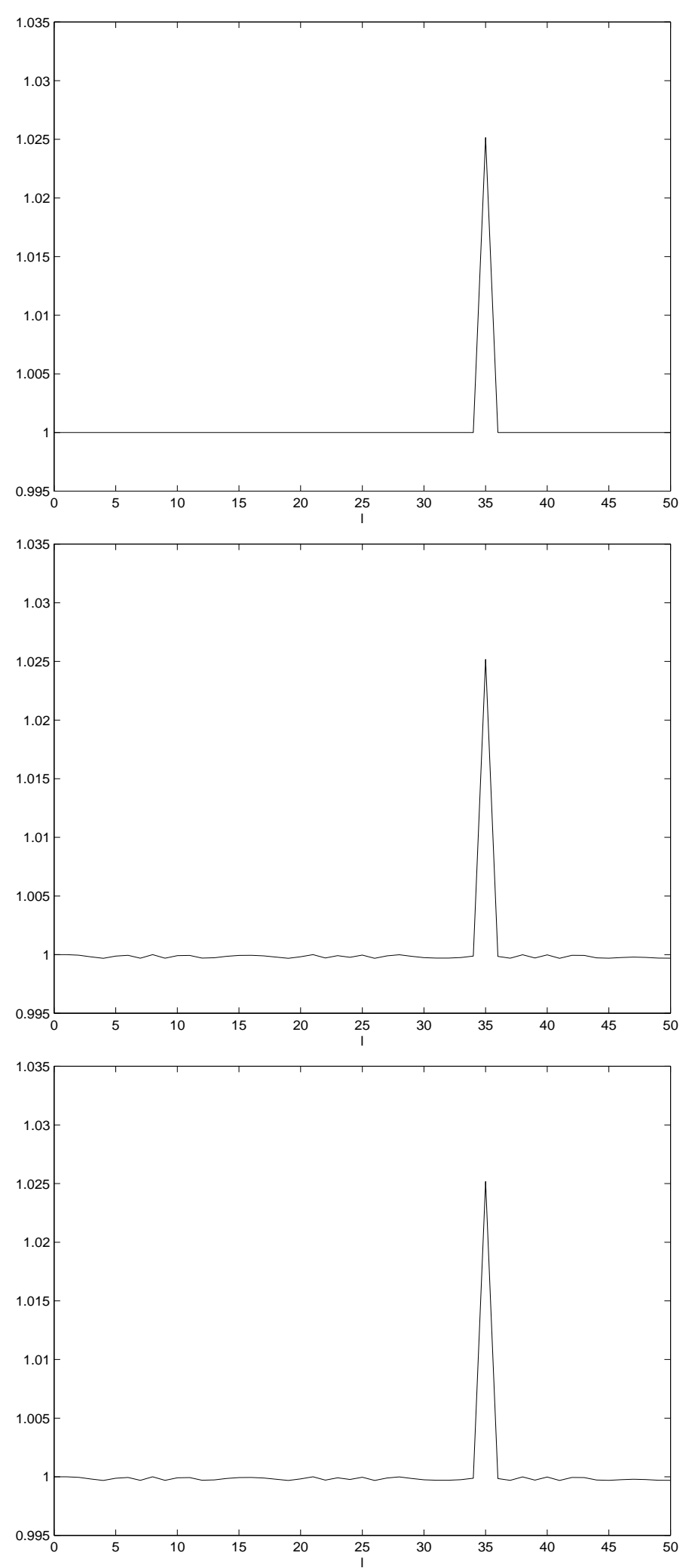

Fig. 5.2. Maximum absolute value of eigenvalues associated to stability matrices: Strang (top) and Lawson method $(\mathrm{d}=1)$ when unprojected (middle) and projected (bottom), when $a=1 / 2, h=0.1$. 

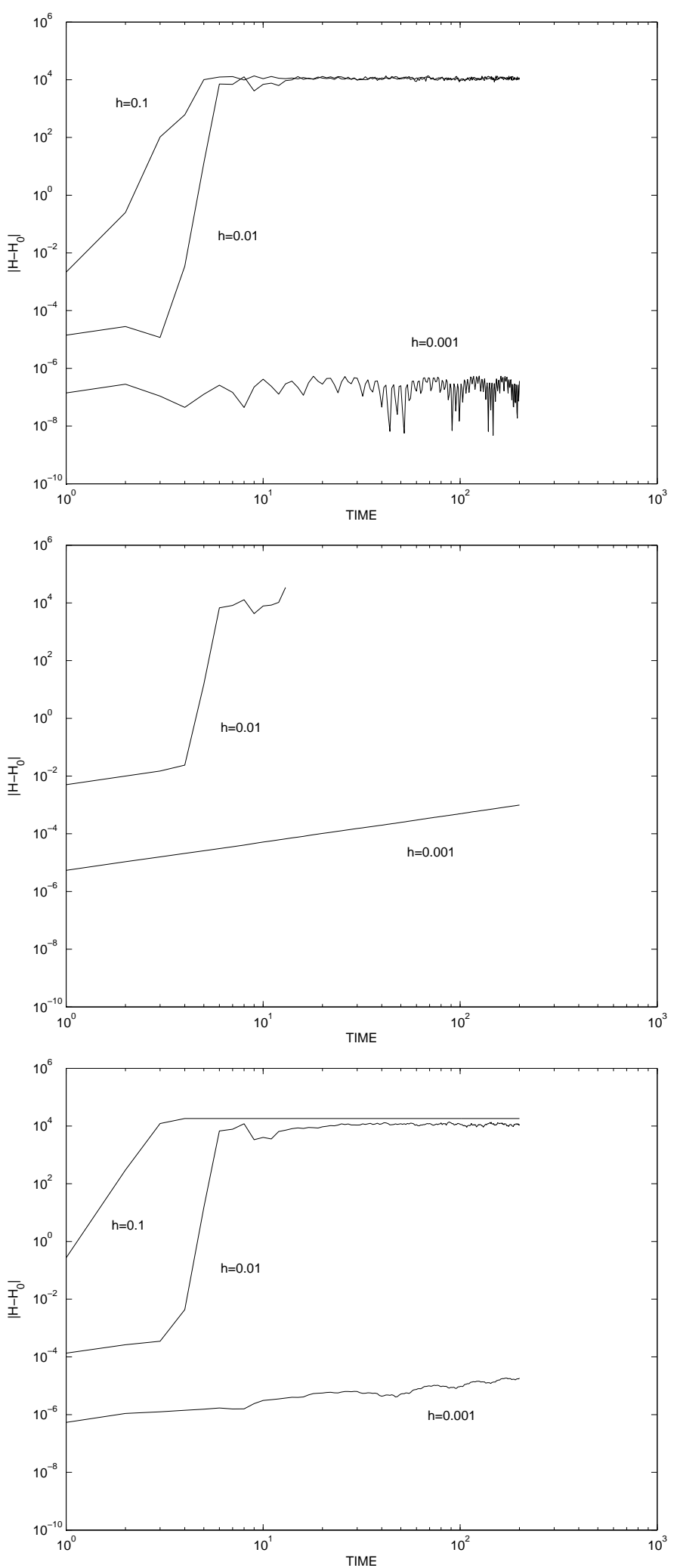

Fig. 5.3. Strang method (top), Lawson method $(\mathrm{d}=1)$ when unprojected (middle) and projected (bottom), when $a=2$. 

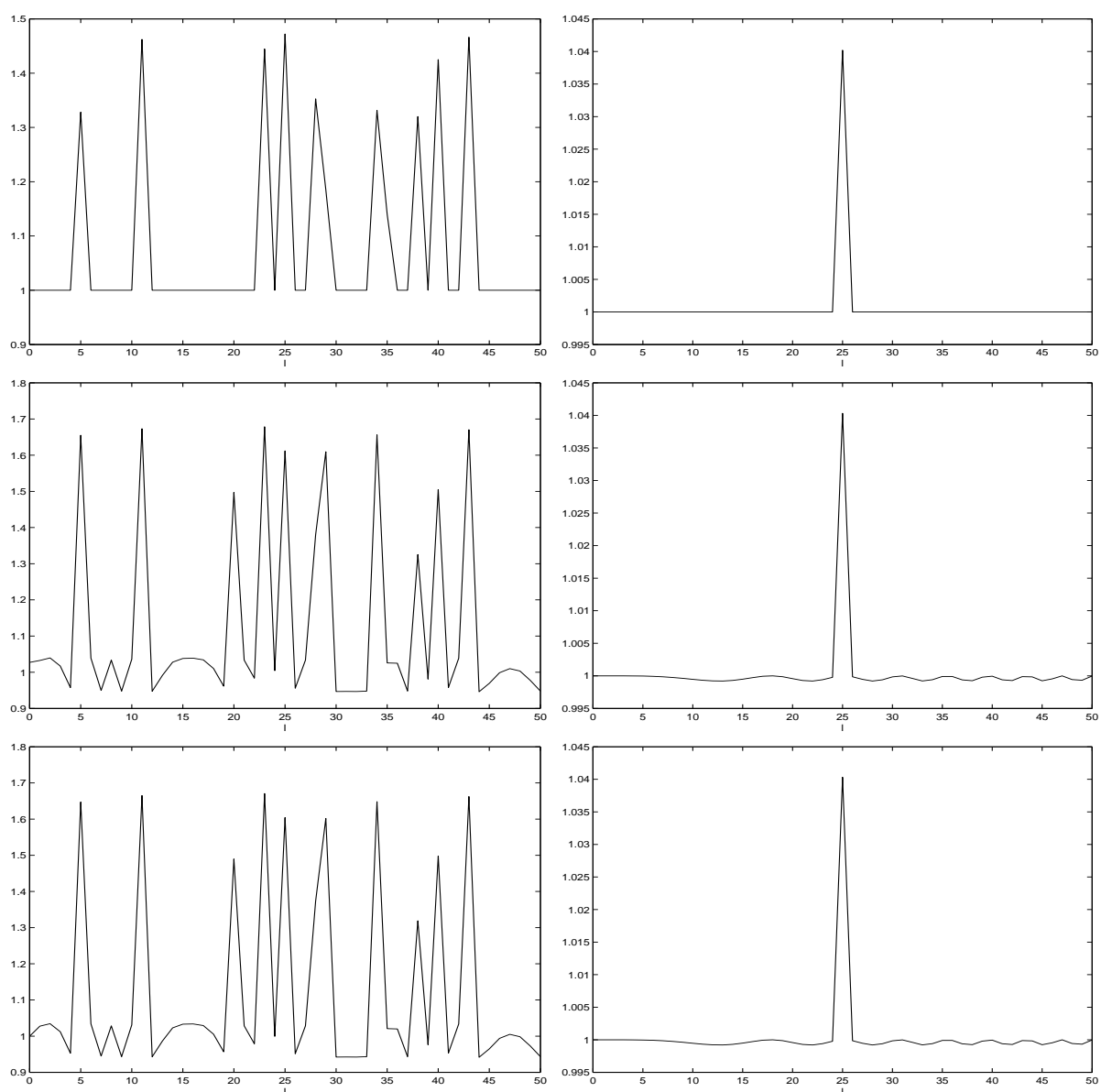

Fig. 5.4. Maximum absolute value of eigenvalues associated to stability matrices: Strang (top) and Lawson method $(\mathrm{d}=1)$ when unprojected (middle) and projected (bottom), when $a=2, h=0.1$ (left) $h=0.01$ (right) and $\lambda=1$. 

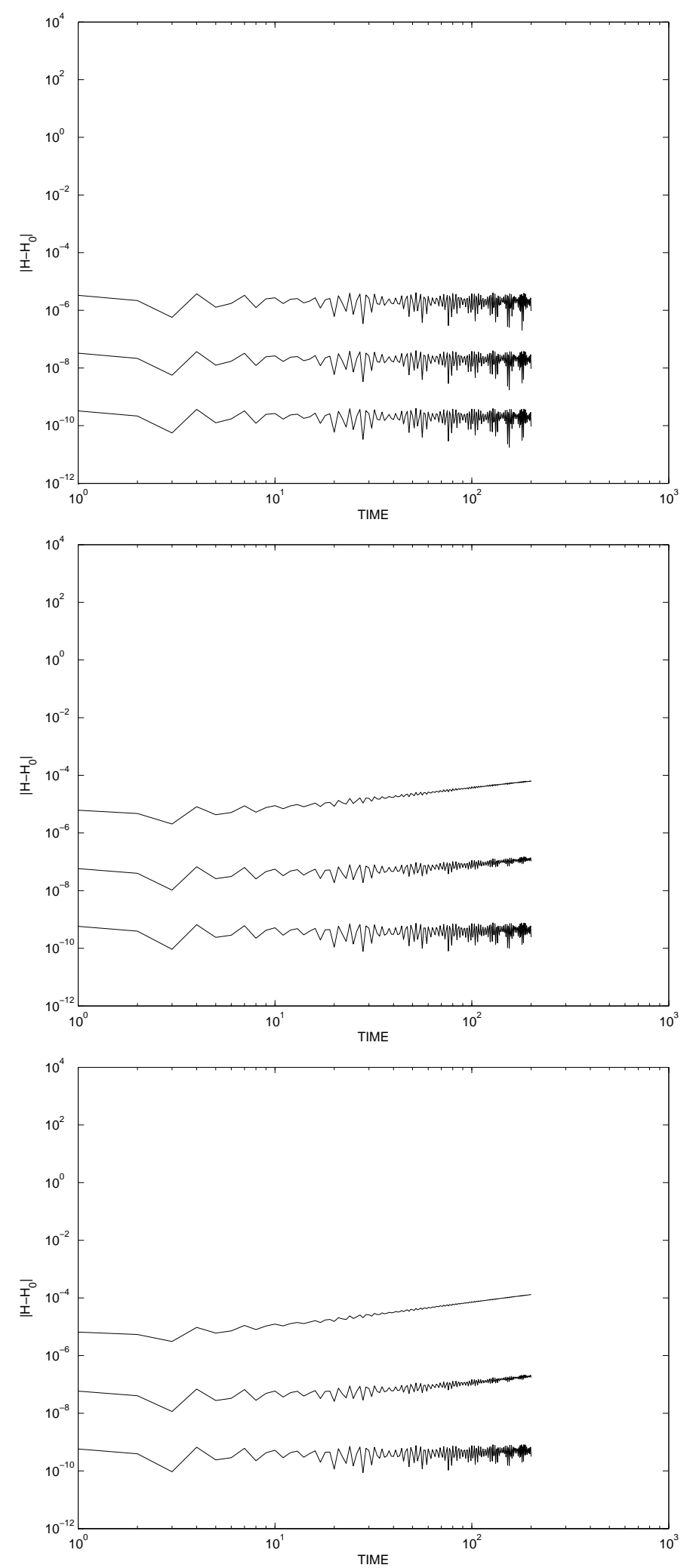

Fig. 5.5. Filtered Strang (top) and filtered Lawson method $(\mathrm{d}=1)$ when unprojected (middle) and unprojected (bottom), when $a=1 / 2$. 

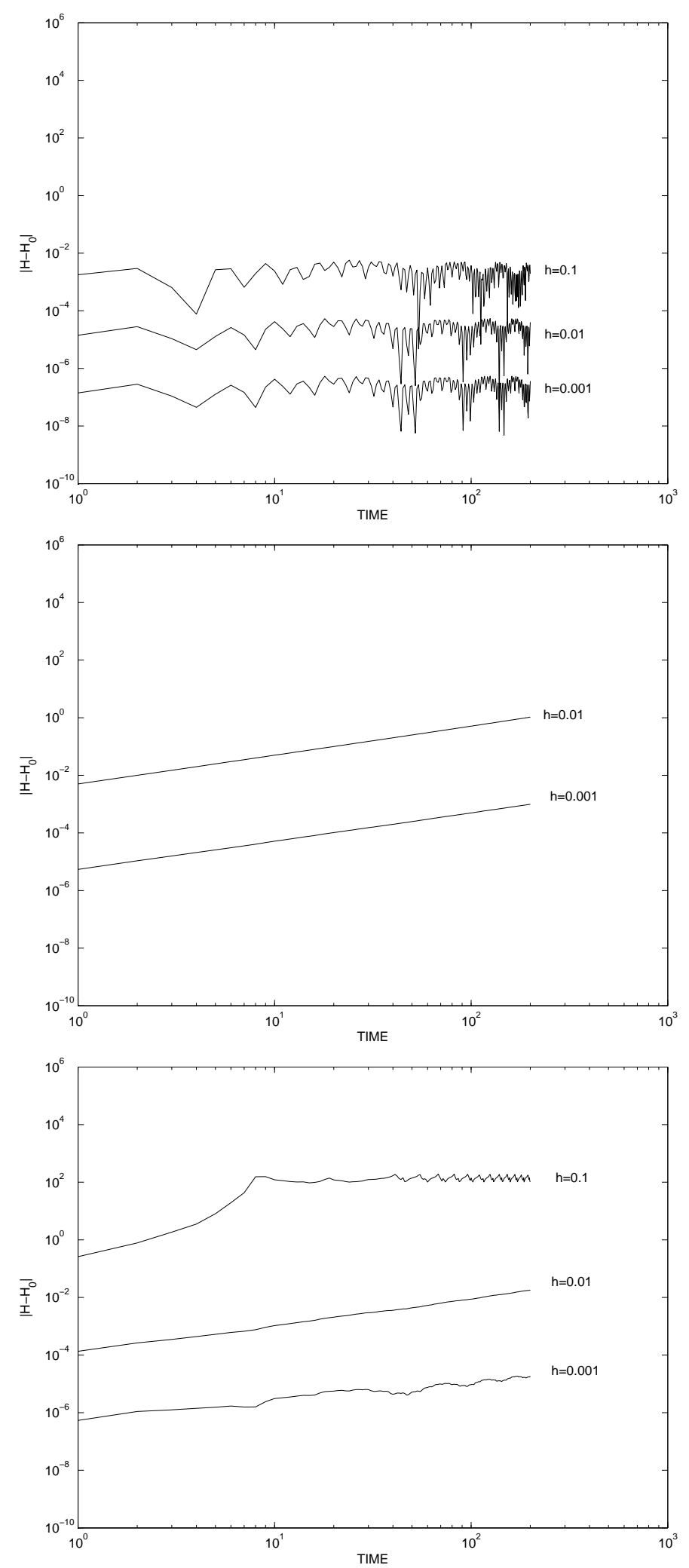

Fig. 5.6. Filtered Strang (top) and filtered Lawson method $(\mathrm{d}=1)$ when unprojected (middle) and unprojected (bottom), when $a=2$. 